\title{
EDITORIAL
}

\section{The hidden hordes}

\author{
With funding for the HMP and Meta-HIT consortia now ending, what's next for these \\ large-scale efforts to map the hidden microbial hordes associated with the human body?
}

If asked, could you immediately point to your left, or indeed your right, retroauricular crease? How about your antecubital fossae? These niches - behind the ears and in the crooks of the elbows, naturally - are just two of the bodily locations examined in an investigation into the structure, function and diversity of the microbiome in healthy humans, which was published recently in Nature $^{1}$. Along with an accompanying framework document ${ }^{2}$, plus 15 other papers published simultaneously in three PLoS journals and available in the PLoS Human Microbiome Project Collection, this represents the first collective major research output from the Human Microbiome Project (HMP) Consortium.

Presciently termed the "second human genome" by David Relman and Stanley Falkow back in 2001 (REF. 3), the microbiome has been one of the hottest topics in microbiology in recent years. The ability to analyse human samples using the culture-independent metagenomic sequencing techniques that were pioneered by environmental microbiologists working in soil and marine environments was demonstrated in 2006 (REF. 4) by the first metagenomic analysis of the gut microbiome in healthy humans. Since then, however, although there have been many fascinating studies on the microbiome, the majority have focused less on its composition and role in maintaining health and more on its influence in diseases such as inflammatory bowel disease and obesity, as well as its role in fundamental processes such as the development and homeostasis of the immune system.

This balance has now begun to be redressed. The HMP study characterized the microorganisms present at up to 18 different body sites on the skin, oral cavity, vagina and nostrils, as well as in faecal samples, for 242 healthy individuals aged $18-40$ and living in two different geographical locations in the United States. The general take-home message from the work, which combined $16 \mathrm{~S}$ rRNA profiling with metagenomic and whole-genome sequencing, is that each individual's microbiome is unique, suggesting that there is no such thing as an overall 'core' human microbiome. Each niche was characterized by one or a few signature taxa and, overall, diversity was greatest in the gut and oral cavity, and least in the vagina. Just over half of the volunteers were sampled at more than one time point, and there appeared to be little variation over time.

Officially launched in 2008, and funded to the tune of $\sim$ US $\$ 150$ million by the US National Institutes of Health
(NIH) Common Fund, the HMP received funding for a 5 -year period, which is now coming to an end. There are, of course, other major consortia working on the microbiome, the largest of which is Meta-HIT (Metagenomics of the Human Intestinal Tract), which, as the name suggests, has focused exclusively on the intestine. Meta-HIT, which involves researchers from the European Union and China, was launched in 2008 and received $€ 22$ million from the European Commission Seventh Framework Programme over a 4-year period, which has also just ended. The main research finding from Meta-HIT was that in European adults the gut microbiota segregates into three main enterotypes ${ }^{5}$, although subsequent studies involving larger sample sizes - including one of the recent HMP papers ${ }^{6}-$ have suggested that this segregation is less discrete and more of a continuum.

The amount of data associated with these projects is staggering. The HMP has generated 3.5 terabytes, more than 1,000 times that generated by the Human Genome Project. But so far the data have raised more questions than answers, and most investigators are clear that there is an urgent need for broader, more multicultural analyses that track individuals over a much longer period of time. Although there is still a definite need for consortia, a combination of this top-down approach with more bottom-up, investigator- and hypothesis-led approaches is the most desirable way forward for many. The $\mathrm{NIH}$ Common Fund office is currently investigating possible funding options for an HMP2, and a first call for proposals in health-related research under the next European Framework Programme is expected in January 2014.

The results of all the microbiome-related research so far have emphasized the pervasive influence of the hidden hordes within. It is to be hoped that, even in these straitened times, the crucial importance of this work will be realized and it will continue to be funded.

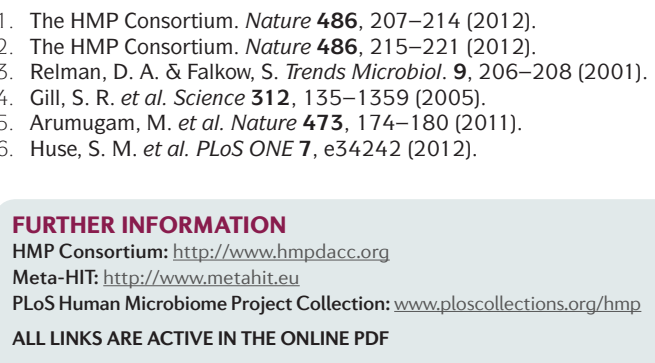

